\title{
A INFLUÊNCIA DO OPEN ACCESS NAS COMUNIDADES ACADÊMICAS DA ÁREA DE BIBLIOTECONOMIA NO NORDESTE DO BRASIL ${ }^{1}$
}

\author{
Virgínia Bárbara de Aguiar Alves \\ Doutora em Ciência da Informação \\ Professora Adjunta do Curso de Biblioteconomia \\ Universidade Federal de Alagoas \\ virginiabarbara@ua.pt \\ Maria Manuel Borges \\ Doutora em Ciências Documentais \\ Professora Auxiliar da Faculdade de Letra \\ Universidade de Coimbra, Portugal \\ mmb@fl.uc.pt
}

\begin{abstract}
Resumo
A pesquisa, que vem sendo desenvolvida, pretende verificar a influência do Open Access, nas comunidades acadêmicas da área de biblioteconomia das Universidades Federais da Região Nordeste do Brasil. O objetivo deste estudo é identificar e discutir a influência do Movimento Open Access (OA), nas comunidades acadêmicas da área de Biblioteconomia das Universidades Federais da Região Nordeste do Brasil. Para a realização da investigação, a metodologia adotada consiste na combinação da abordagem qualitativa e da quantitativa. Os instrumentos de recolha de dados utilizados foram o questionário e a entrevista. Os questionários foram aplicados aos professores e alunos dos cursos de Biblioteconomia, com a finalidade de verificar os efeitos e as consequências do movimento sobre estas comunidades, visando responder à seguinte questão: Quais os efeitos/consequências que a "via verde" e a "via dourada" de disseminação da informação trazem para a comunicação científica? Os resultados do inquérito aplicado aos professores dos cursos de Biblioteconomia da Região Nordeste do Brasil que serão abordados neste trabalho, mostram claramente o impacto do OA no Brasil e, de uma forma muito especifica, nas comunidades acadêmicas da área de Biblioteconomia no Nordeste do Brasil.
\end{abstract}

\section{Palavras-chave}

Open Access. Acesso livre. Open Access - influência. Acesso livre (Open Access) - Região Nordeste do Brasil.

\footnotetext{
${ }^{1}$ Este trabalho resulta da investigação para um programa doutoral (ICPD - Universidade de Aveiro e Universidade do Porto) co-orientado pela co-autora, Prof. ${ }^{a}$ Doutora Maria Manuel Borges da Universidade de Coimbra e orientado pela Prof. ${ }^{a}$ Doutora Fernanda Ribeiro da Universidade do Porto.
} 


\section{INTRODUÇÃO}

A procura da informação e a sua disseminação atravessou gerações e, entre o final do século XX e o início do século XXI surgiu o movimento do Open Access (OA), um modelo alternativo para a comunicação científica, que vem contribuindo para a consolidação dessa aspiração. $\mathrm{O}$ novo modelo estabeleceu padrões para a interoperabilidade entre bibliotecas digitais e repositórios temáticos e institucionais, desencadeando uma verdadeira rede de eventos em todo o mundo e também no Brasil, com o objetivo de apoiar o movimento para o acesso livre ao conhecimento científico e entre os quais podemos citar:

- a Declaração Sobre a Ciência no Século XXI (1999) e a Budapest Open Access Initiative (2002);

- a Reunião promovida pelo Open Society Institute (OSI), a qual estabeleceu duas estratégias - a via verde (auto-arquivamento em repositórios institucionais e temáticos dos artigos publicados em revistas, que não são de acesso livre, mas que podem ser acedidas com a autorização da revista) e a via dourada (publicação em revistas de acesso livre);

- a Bethesda Statement on Open Access Publishing (2003), visando estabelecer princípios para obter apoio formal das agências de financiamento e de todos os atores do fluxo da comunicação científica para a publicação de resultados de pesquisa científica;

- a Berlin Declaration on Open Access to Knowledge in Sciencies \& Humanities (2003) que recomenda o uso consistente da Internet para divulgação e publicação das pesquisas científicas, encorajando os pesquisadores a publicarem os seus trabalhos em revistas de acesso livre, além de endossar as declarações anteriores;

- a IFLA (International Federation of Library Associations) on Open Access for Scholarly Literature (2003) - que afirma que abrir o acesso à literatura científica e a resultados de pesquisa é fundamental para compreensão do mundo e para identificação de solução aos desafios globais e, em particular a redução da desigualdade de informação;

- a Declaração de Salvador Para Acesso Aberto (2005) - conclama a todos os parceiros da comunidade científica internacional para, conjuntamente, assegurar que a informação científica seja de livre acesso e disponível para todos e, finalmente;

- o Manifesto Brasileiro de apoio ao Acesso Livre à Informação Científica (2005) - chama a atenção da comunidade científica brasileira para apoiar o movimento mundial em favor do acesso livre à informação científica.

O movimento do acesso livre (OA) vem buscando tornar possível a comunicação científica entre milhares de pesquisadores espalhados pelo mundo, fragmentando assim as barreiras geográficas e facilitando a disseminação da literatura científica, constituindo-se como recurso fundamental para a construção do conhecimento, que compreende a produção, a comunicação (disseminação do conhecimento) e a utilização do conhecimento produzido na criação de novos conhecimentos.

$O$ acesso à informação tem-se mostrado como um enorme desafio, principalmente quando se trata da informação científica. Com a crise dos periódicos e com o elevado custo na manutenção das assinaturas das revistas científicas, o acesso fica bastante reduzido. Face a este fato, atualmente, são discutidos novos meios e novos procedimentos de cooperação entre sistemas de informação, viabilizados a partir do surgimento de normas de interoperabilidade, com a finalidade de disponibilizar textos completos diretamente na Internet (teses, artigos de periódicos, trabalhos em congressos, literatura cinzenta), para que se tornem visíveis e acessíveis nacional e internacionalmente.

\section{OBJETIVOS E METODOLOGIA}

A pesquisa que apresentamos tem como objetivo identificar e discutir a influência do movimento $\mathrm{OA}$ nas 
comunidades acadêmicas da área de Biblioteconomia das Universidades Federais da Região Nordeste do Brasil, com ênfase sobre as questões relativas à "via verde" e à "via dourada". As duas vias enquanto estratégias básicas do Movimento de Acesso Livre à informação científica foram estabelecidas com a finalidade de acelerar a disseminação do conhecimento científico. Pretendemos, pois, saber quais os efeitos/consequências que a via verde e a via dourada de disseminação da informação trazem para a comunicação científica na Região Nordeste do Brasil, mais precisamente identificar e discutir a influência do $\mathrm{OA}$ nas comunidades acadêmicas da área de Biblioteconomia nas Universidades Federais da Região Nordeste do Brasil. Nesse sentido, a metodologia empregue na construção desta investigação envolve a recolha de dados, nomeadamente, através de entrevistas e questionários.

O questionário foi elaborado com perguntas de identificação (idade, gênero, profissão e habilitações acadêmicas); perguntas de informação, com a finalidade de colher dados sobre fatos e opiniões; perguntas de descanso ou de preparação que servem para introduzir uma pausa ou mudar de assunto e introduzir perguntas com maior grau de dificuldade; e, finalmente, perguntas de controle para verificar a autenticidade de outras, contendo, na medida do possível, perguntas fechadas, com apresentação do tema a ser investigado e a apresentação do pesquisador com instruções precisas, claras e curtas sobre como respondê-lo (CARMO; FERREIRA, 2008, p. 154).

\section{DISCUSSÃO E RESULTADOS}

O movimento do acesso livre a informação científica consiste no resultado de uma longa história de comunicação acadêmica, que sempre esteve ligada às mudanças tecnológicas e econômicas. Artigos científicos e monografias têm estado implicados nas recentes mudanças (SWAN, 2006, p. 1).

O Acesso Livre significa a disponibilização livre na Internet de literatura de carácter académico ou científico, permitindo a qualquer utilizador ler, descarregar, copiar, distribuir, imprimir, pesquisar ou referenciar o texto integral desses documentos. Ao contrário de outros autores, os investigadores e académicos publicam os resultados do seu trabalho não para obterem rendimentos (direitos de autor, etc.), mas para obterem outro tipo de recompensa. Os investigadores são recompensados (progressão na carreira, financiamento dos seus projectos, prémios científicos, etc.) pela sua produtividade científica, que é avaliada não apenas pela sua dimensão (quantidade), mas sobretudo pelo seu impacto (qualidade), usualmente associado ao número de citações (RODRIGUES, 2006).

\section{E para Tarragó (2007) com o} movimento do acesso livre (OA), todos os atores envolvidos no sistema de comunicação recebem beneficios e entre os quais podemos citar insvestigadores/autores, leitores, professores e estudantes, bibliotecas, universidades, revistas e editoras, agências de fomentos, governo e cidadãos.

Se houve um tempo em que editores científicos tiveram um papel incontornável na divulgação do conhecimento, constitui hoje, pela total arbitrariedade de custos dos títulos que oferecem, os principais obstáculos a essa mesma difusão que resulta da incapacidade de suportes pelas instituições e respectivas bibliotecas (BORGES, 2006, p. 72).

A comunicação da ciência ou a comunicação científica online tornou-se uma atividade indispensável para o mundo acadêmico: "O acesso aberto garante novas perspectivas aos pesquisadores, ao permitir e facilitar a divulgação científica, o acesso à informação produzida em escala mundial, acelerando assim o impacto dos estudos" (CRESPO; CORREIA, 2006). 
Na perspectiva de Batista et al (2007, p. 2), o Acesso Livre, como já vem sendo bastante citado na literatura, é concomitantemente o resultado de uma negação dos pesquisadores ao modelo de negócios de editoras comerciais de revistas científicas e de seus preços cada vez mais altos por inviabilizarem o acesso à informação e com isso terem impacto negativo no impacto de citação que daí pode resultar. O propósito do movimento mundial em favor do Acesso Livre a resultados de pesquisa é a disseminação ampla e irrestrita dos resultados de pesquisas custeadas com recursos públicos.

Para Mueller (2006, p. 1), o movimento para o acesso livre ao conhecimento científico pode ser considerado o evento mais significativo da nossa época, no que se refere à comunicação científica, e, neste sentido, se torna um grande desafio para a comunidade académica. Quanto mais sucesso obtiver o movimento, maior será o impacto sob as mudanças provocadas no sistema tradicional de comunicação cientifica.

O Brasil vem sobressaindo quanto às políticas de acesso a informação. Tem realizado diferentes iniciativas em favor do acesso livre. De acordo com Melo, Sampaio e Pires (2007) o Brasil, devido à dimensão do seu território e, consequentemente, às desigualdades regionais, não se desenvolve de maneira semelhante nas diversas regiões, pelo que especificamente no contexto educativo, ainda convive com diferenças na educação das classes mais abastadas, por um lado, e das classes menos favorecidas, por outro lado. Neste seguimento, os autores supracitados destacam o impacto das diferenças regionais e do investimento na disseminação do conhecimento, defendendo que as regiões que possuem mais conhecimento podem partilhar os seus recursos informacionais com as regiões menos beneficiadas, cooperando para o desenvolvimento geral da nação.

A partir de 2005, com a elaboração Manifesto Brasileiro de apoio ao Acesso Livre à Informação Científica, e segundo Kuramoto (2008), o Brasil vem realizando através do IBICT, várias inciativas em favor desse movimento entre as quais podemos mencionar: a assinatura da Declaração de Berlim (2003), a submissão, a aprovação e o desenvolvimento do Projeto de Publicações Periódicas de Acesso Livre (PCAL), junto a Financiadora de Estudos e Projetos (FINEP), a prospecção, a identificação, a absorção, a customização, e o aperfeiçoamento e a distribuição de tecnologias para o tratamento e disseminação da informação, que dão suporte às ações de acesso livre a exemplo do Sistema Eletrônico de Editoração de Revistas (SEER), a construção de repositórios institucionais e temáticos de acesso livre, Projeto de Lei (PL) 1120/2007 que dispõe sobre o processo de disseminação da produção científica, a implantação da Biblioteca Digital Brasileira de Teses e Dissertações (BDTD). Para além destas iniciativas, foram publicados, em 2006, um número especial da revista Ciência da Informação (35:2), e, em 2008, da Revista LIINC (4:2) ambas com artigos de pesquisadores brasileiros e estrangeiros sobre o tema do acesso livre.

Além das iniciativas acima citadas, outras declarações de apoio ao OA vêm sendo elaboradas por instituições e/ou associações acadêmicas em várias partes do Brasil como a Declaração de Salvador (2005), a Carta de São Paulo (2005), a Carta de Florionópolis (2006), a Carta aberta elaborada durante a $58^{\mathrm{a}}$ reunião anual da Sociedade Brasileira para o Progresso da Ciência (2006), a Carta do Rio de janeiro (2007) e a Carta de Apoio ao Acesso Aberto à Produção Técnico-Científica, Artística e Didática da Universidade de São Paulo (2010).

Para Kuchma (2008), as declarações de acesso livre, nos países em desenvolvimento, encorajam a cooperação regional, como a Declaração para o Acesso Livre de Salvador (Salvador Declaration on Open Access) - uma perspectiva do mundo em desenvolvimento (Developing World Perspective), redigida em 2005, na Bahia, Brasil; e, uma política Nacional do Acesso Livre em Países em Desenvolvimento 
(National Open Access Policy for Developing Countries), redigida em 2006 na Índia no Workshop em Edição Eletrônica e Acesso Livre (Workshop on Electronic Publishing and Open Access). Um dos exemplos mais bemsucedidos de cooperação regional é o consórcio Scientific Electronic Library Online (Scielo).

E ressaltamos também a participação brasileiara na Iniciativa ALEMPLUS (2006), no Compromisso do Minho (2006) e a Cooperação Luso-brasileira (2009). Esta última, foi realizada coma finalidade de aumentar a difusão do conhecimento científico em língua portuguesa, nos países lusófonos e no mundo.

A pesquisa realizada procurou identificar os efeitos e/ou as consequências desse movimento sobre a comunidade acadêmica da area de Biblioteconomia na Região Nordeste do Brasil e como podemos verificar, através dos indicativos apresentados nos primeiros resultados recolhidos, nomeadamente do inquérito aplicado aos professores dos cursos de Biblioteconomia da Região Nordeste do Brasil, é possível concluir o impacto do OA nas comunidades acadêmicas da área de Biblioteconomia no Nordeste do Brasil. E em um universo de aproximadamente cento e trinta e um professores cinquenta e um responderam a pesquisa.

Uma das primeiras conclusões retiradas respeita à implantação de repositórios institucionais. A maioria dos inquiridos $(67,3 \%)$ afirma existir repositório institucional na universidade onde trabalha.

Tabela 1 - Implantação de repositórios institucionais

\begin{tabular}{|l|c|c|}
$\begin{array}{l}\text { A universidade onde } \\
\text { trabalha possui }\end{array}$ & $\begin{array}{c}\text { Frequência } \\
\text { (N) }\end{array}$ & $\begin{array}{c}\text { Percentual } \\
(\%)\end{array}$ \\
$\begin{array}{l}\text { Repositório } \\
\text { Institucional }\end{array}$ & & \\
\hline Sim & 33 & $67,3 \%$ \\
\hline Não & 16 & $32,7 \%$ \\
\hline
\end{tabular}

Fonte: Autora, 2013.

Constatámos, talvez pelo facto de os repositórios terem esta implantação, que a maioria dos professores inquiridos $(85,1 \%)$ utilizam repositórios institucionais como fonte de pesquisa para realização dos seus trabalhos acadêmicos e apenas (14,9\%) ainda não utilizam repositórios institucionais como fonte de pesquisa para realização de seus trabalhos acadêmicos conforme apresentamos na tabela abaixo.

Tabela 2 - Utilização de repositórios institucionais para pesquisa

\begin{tabular}{|l|c|c|}
\hline $\begin{array}{l}\text { Utiliza Repositório } \\
\text { Institucional como } \\
\text { fonte de pesquisa }\end{array}$ & $\begin{array}{c}\text { Frequência } \\
\text { (N) }\end{array}$ & $\begin{array}{c}\text { Percentual } \\
(\mathbf{\%})\end{array}$ \\
\hline Sim & 40 & $85,1 \%$ \\
\hline Não & 7 & $14,9 \%$ \\
\hline
\end{tabular}

Fonte: Autora, 2013.

Outro aspecto relevante é a utilização da produção bibliográfica em OA e $\mathrm{O}$ seu impacto nas comunidades académicas. $\mathrm{Na}$ verdade, a maioria dos professores inquiridos $(90,0 \%)$ utiliza produção bibliográfica em OA para realização de seus trabalhos académicos de acordo com a tabela abaixo.

Tabela 3 - Utilização de produção bibliográfica em OA

\begin{tabular}{|l|c|c|}
$\begin{array}{l}\text { Utiliza produção } \\
\text { bibliográfica em }\end{array}$ & $\begin{array}{c}\text { Frequência } \\
\text { (N) }\end{array}$ & $\begin{array}{c}\text { Percentual } \\
\text { OA } \%)\end{array}$ \\
\hline Sim & 36 & $90,0 \%$ \\
\hline Não & 4 & $10,0 \%$ \\
\hline
\end{tabular}

Fonte: Autora, 2013.

Ainda confirmamos através da pesquisa outro impacto significativo com relação a produção bibliográfica que a maioria dos professores inquiridos $(74,3 \%)$ possuem produção bibliográfica em OA tal como apresentamos na tabela seguinte.

Tabela 4 - Produção bibliográfica em OA

\begin{tabular}{|l|c|c|}
\hline $\begin{array}{l}\text { Possui } \\
\text { produção } \\
\text { bibliográfica em }\end{array}$ & $\begin{array}{c}\text { Frequências } \\
\text { (N) }\end{array}$ & $\begin{array}{c}\text { Percentual } \\
\text { (\%) }\end{array}$ \\
\hline Sim & 26 & $74,3 \%$ \\
\hline Não & 9 & $25,7 \%$ \\
\hline
\end{tabular}

Fonte: Autora, 2013. 
Asseguramos através da pesquisa outro fator de impacto bastante interessante em favor do movimento OA, que a maior parte dos professores inquiridos $(98,0 \%)$ utilizam periódicos eletrônicos em $\mathrm{OA}$ como fonte de pesquisa para realização de seus trabalhos acadêmicos.

Tabela 5 - Utilização de periódicos eletrônicos

\begin{tabular}{|l|c|c|}
\hline $\begin{array}{l}\text { Utiliza periódicos } \\
\text { eletrônicos como fonte } \\
\text { de pesquisa }\end{array}$ & $\begin{array}{c}\text { Frequência } \\
(\mathbf{N})\end{array}$ & $\begin{array}{c}\text { Percentual } \\
\mathbf{( \% )}\end{array}$ \\
\hline Sim & 48 & $98,0 \%$ \\
\hline Não & 1 & $2,0 \%$ \\
\hline Fonte: Autora, 2013. & & \\
\hline
\end{tabular}

E como demonstra a tabela seguinte, podemos constatar mais um indicador positivo do movimento OA no Brasil: uma grande parte dos professores inquiridos $(83,3 \%)$ conhecem o movimento do OA.

Tabela 6 - Conhecimento do OA

\begin{tabular}{|l|c|c|}
\hline $\begin{array}{l}\text { Conhecimento } \\
\text { do AO }\end{array}$ & $\begin{array}{c}\text { Frequência } \\
\text { (N) }\end{array}$ & $\begin{array}{c}\text { Percentual } \\
(\mathbf{\%})\end{array}$ \\
\hline Sim & 40 & $83,3 \%$ \\
\hline Não & 8 & $16,7 \%$ \\
\hline Fonte: Autora, 2013. & &
\end{tabular}

Para corroborar esta abertura, a maioria dos inquiridos $(97,5 \%)$ concordam com o movimento do OA.
Tabela 7 - Concordância com o movimento OA

\begin{tabular}{|l|c|c|}
\hline $\begin{array}{l}\text { Concorda com o } \\
\text { Movimento do OA }\end{array}$ & $\begin{array}{c}\text { Frequência } \\
\text { (N) }\end{array}$ & $\begin{array}{c}\text { Percentual } \\
\text { (\%) }\end{array}$ \\
\hline Sim & 39 & $97,5 \%$ \\
\hline Não & 1 & $2,5 \%$ \\
\hline
\end{tabular}

Fonte: Autora, 2013.

Apresentámos alguns resultados do inquérito aplicado aos professores dos cursos de Biblioteconomia da Região Nordeste do Brasil, mas já podemos identificar determinados efeitos/consequências do movimento do OA. Podemos afirmar que a comunidade acadêmica da área de biblioteconomia, concorda e aceita o acesso livre, e que já se torna visível o impacto ou a influência do movimento do acesso livre no Brasil, e, especificamente, na região nordeste. Contudo, dever-se-á continuar a investir na divulgação do movimento em prol do acesso livre e a implementar programas e projetos, de forma a ampliar e estimular a utilização do auto-arquivamento (via verde) e a criação de periódicos de acesso livre (via dourada). Este impulso não deverá ser proporcionado só através governo brasileiro, só através do IBCT, mas também através do envolvimento direto das próprias comunidades acadêmicas com os seus pares, nas suas instituições, para que o acesso livre se torne de fato uma alternativa para a disseminação do conhecimento científico e para que o Brasil e especificamente a Região Nordeste tenham a possibilidade de aumentar a visibilidade da sua produção científica no Brasil e no mundo.

\title{
THE INFLUENCE OF THE OPEN ACCESS ON THE ACADEMIC COMMUNITIES OF THE BIBLIOTHECONOMY'S AREA IN THE NORTHEASTERN REGION OF BRAZIL
}

\begin{abstract}
The research developed had the purpose to verify the influence of the Open Access in library and information science community of the Federal Universities of the Northeast Region of Brazil in order to answer the following question: What are the effects/consequences that the "green road" and the "golden road" of dissemination of information bring to scientific communication? To this purpose, the method applied combined the qualitative and quantitative approaches. To collect data we used questionnaires and interviews. In this article, we will show the results of the inquiry applied to teachers of library and information science courses from the Northeast Region of Brazil that will be approached in this work show clearly the impact of the $O A$ in Brazil and, in a very specific way, in the academic communities mentioned above.
\end{abstract}


A Influência do Open Access nas Comunidades Acadêmicas da Área de Biblioteconomia no Nordeste do Brasil

\author{
Keywords \\ Open access. Free Access. Open Access - influence. Open Access (Open Access) Northeastern Region of Brazil.
}

Artigo recebido em 25/11/2013 e aceito para publicação em 11/03/2014

\section{REFERÊNCIAS}

BAPTISTA, A. A. et al. Comunicação científica: o papel da open archives initiative no contexto do acesso livre. Enc. Bibli: R. Eletr. Bibliotecon. Ci. Inf., $1^{\circ}$ sem. 2007, p. 5-17. Disponível em:

$<$ http://reposiemtorium.sdum.uminho.pt/bits tream/1822/8727/1/2007EncontrosBibli.pdf >. Acesso em: 11 nov. 2010.

BORGES, M. M. A esfera: comunicação acadêmica e novos mídia. 2006. Tese

(Doutorado em Letras) - Faculdade de Letras, Universidade de Coimbra, Coimbra, Portugal.

CARMO, H.; FERREIRA, M. M.

Metodologia da investigação: guia para auto-aprendizagem. Lisboa: Universidade Aberta, 2008.

CRESPO, I. M.; CORREIA, Cyntia H. W. Acesso livre à comunicação científica: a experiência do Scielo. Revista F@ro,n. 6, p. 1-6. Disponível em:

$<$ http://web.upla.cl/revistafaro/03_estudios/ 06_index.htm>. Acesso em: 6 jan. 2009.

KUCHMA, I. Open Access, Equity, and Strong Economy in Developing and trasition countries: policy perspective. Serials Review, n. 1, p. 13-20. Disponível em:

$<$ http://www.sciencedirect.com/science?_ob =ArticleURL\&_udi=B6W63

4RTKMRW1\&_user=2459663\&_rdoc $=1 \& \_f$ $\mathrm{mt}=\& \_o r i g=$ search\&_sort $=\mathrm{d} \& v i e w=\mathrm{c} \& \_a c c t$ $=$ C000057389\&_version $=1 \& \_u r l V e r s i o n=0 \&$ _userid $=2459663 \& \mathrm{md} 5=\mathrm{b} 0 \mathrm{c} 320 \mathrm{c} 27 \mathrm{c} 00 \mathrm{~b} 05 \mathrm{a} 96$ 0e02fe206452c8>. Acesso em: 18 maio 2008.
MELO, L. B; SAMPAIO, M. I. C.; PIRES, C. A questão do acesso aberto em Portugal e no Brasil. In: SEMINÁRIO NACIONAL DE BIBLIOTECAS UNIVERSITÁRIAS, 15, São Paulo, 2008. Anais eletrônicos... São Paulo: SNBU, 2008, p. ?-?. Disponível em: <http://www.sbu.unicamp.br/snbu2008/anai s/site/pdfs/2796.pdf $>$. Acesso em: 12 nov. 2009.

MUELLER, S. P. M. A comunicação científica e o movimento de acesso livre ao conhecimento. Ciência da Informação, n. 2, p. 27-38. Disponível em:

$<$ http://www.scielo.br/pdf/ci/v35n2/a04v35 n2.pdf $>$. Acesso em: 30 out. 2006.

RODRIGUES, E. Acesso Livre: utopia ou realidades. In: ENCONTRO NACIONAL DE BIBLIOTECAS JURÍDICAS, 1, Lisboa, 2004 - "Direito e informação: actas". Anais... Coimbra: Coimbra, 2006, p. 29-40. ISSN 08703116. Disponível em:

<http://repositorium.sdum.uminho.pt/handle /1822/4942>. Acesso em: 12 dez. 2009.

SWAN, A.; GUÉDON, J. Open access história, definitions and rationale In: JACOBS, N. Open Access: key strategic, technical and economic aspects (Parte1). England: Chandos, 2006

TARRAGÓ, N. S. El movimiento de acceso abierto a la información y las políticas nacionales e institucionales de autoarchivo. ACIMED, n. 3. Disponível em: <http://scielo.sld.cu/pdf/aci/v16n3/aci05907 .pdf>. Acesso em: 20 maio 2009.

KURAMOTO, H. Acesso livre à informação científica: novos desafios. Liinc em Revista, n. 2, p. 154-157. Disponível em: $<$ http://www.ibict.br/liinc>. Acesso em: 12 set 2008 . 